\title{
Measurement Reconstruction in Sensor Networks for Industrial Systems
}

\author{
Yu Zhang, Chris Bingham, Sepehr Maleki
}

\begin{abstract}
For signal processing in sensor networks there is an on-going challenge for filling missing information when it is either incomplete, uncertain or biased, in ways that are both efficient and with confidence. This paper reviews three established and additional newly developed techniques addressing the problem. Considering sensor signals that are highly correlated in a sensor network, one sensor measurement can be reconstructed based on measurements from other sensors. In such cases, three signal reconstruction methods are considered: 1) principal component analysis (PCA) based missing value approach; 2) self-organizing map neural network (SOMNN) based algorithm; and 3) an analytical optimization (AO) technique. To demonstrate the efficacy of the methods, temperature data are studied on an industrial gas turbine system, where, especially, a faulty sensor signal is utilized to be reconstructed from the other sensor measurements.
\end{abstract}

Keywords - signal reconstruction, principal component analysis, self-organizing neural network, analytical optimization.

\section{Introduction}

Electronic equipment now supports almost every technical device and appliance to help a user or operator, with sensors taking the role of localised 'eyes and ears'. To retrieve reliable and accurate information from sensor networks is of special importance in complex systems due to the benefits of reducing down-time and loss of productivity, and increasing the assurance of safety, quality and reliability of systems [1].

Following identification of a faulted sensor, a decision needs to be made as to whether operation of the unit can continue, possibly at reduced capacity or lower performance, or whether the unit should be shut-down for immediate maintenance. The latter option is often considered a significant disturbance with enforced downtime leading to lost productivity. An alternative, therefore, is to try and reconstruct a 'best estimate' of the measurements expected from the faulted sensor with a view to facilitate improved unit availability [2].

Principal component analysis (PCA) is extensively applied for data analysis purposes to reduce a large dataset whilst still preserving 'sufficient' information contained in the original data. The PCA-based missing value approach is used to reconstruct the faulted signal by using measurements from non-faulted sensors and the correlations established from training data. [3]
Artificial neural networks (ANNs) have been applied extensively for system identification based on black-box modeling through analytical means, especially successfully for highly nonlinear complex dynamical systems. Based on self-organizing map neural network (SOMNN) algorithm, for a 2-dimensional output space, the faulted signal can be reconstructed by adjusting the weight vector using a combination of its $k$-nearest nodes. [4]

An analytical optimization (AO) technique has been presented in [5] to use a generalized multi-dimensional linear regression technique to provide a general optimal affine linear transform to facilitate signal reconstruction in the event of sensor failure by solving a $l_{2}$-norm minimization problem, for sensor fault detection and sensor measurement reconstruction, and a minimal sensor set can be selected based on redundant information.

In this paper, the three approaches are applied for sensor measurement reconstruction in an industrial gas turbine system. The relative merits of each method is discussed through use of experimental trials.

\section{Methodology}

Here, the treatment is restricted to a brief overview to introduce terminology and definitions that are subsequently used for the three signal reconstruction approaches.

\section{A. $\quad$ PCA}

Let $\boldsymbol{X}$ be the original data matrix, with $I$ rows indicating the sensor samples and $J$ columns of time steps. The covariance matrix $\boldsymbol{C}$ is then defined by

$$
C=\frac{1}{J} \sum X X^{T}
$$

The eigenvectors and eigenvalues of the covariance matrix are found from

$$
\boldsymbol{V}^{-1} \boldsymbol{C} \boldsymbol{V}=\boldsymbol{\Lambda}
$$

The eigenvectors and eigenvalues are rearranged in order of decreasing eigenvalues. The first $P$ columns of $\boldsymbol{V}$ are then retained as the basis matrix $V_{\alpha}$, where $1 \leq P \leq I$.

To describe the original data in principal component space, the following relation is used:

$$
\boldsymbol{Y}=\boldsymbol{V}_{\alpha}^{T} \boldsymbol{X}
$$

where $\boldsymbol{Y}$ is the principal component matrix, which is a representation of $\boldsymbol{X}$ after PCA, with the $i$ th row representing the $i$ th principal component [6]. 


\section{International Journal of Advances in Computer Science \& Its Applications}

Volume 6 : Issue 1 [ISSN 2250-3765]

Publication Date : 18 April, 2016

The PCA based missing value approach is used to reconstruct the signal by using the measurements from the other sensors and the correlations of the sensors from the training data. Assume the $i$ th sensor is faulty, and the input signal is the original signal without the $i$ th term

$$
\boldsymbol{x}_{i}=\left[\boldsymbol{x}_{-i}^{T} \boldsymbol{x}_{+i}^{T}\right]^{T} .
$$

The eigenvector matrix $\boldsymbol{V}_{\alpha}$ is modified by eliminating the $i$ th row which the $i$ th sensor contributed to:

$$
\boldsymbol{V}_{i}=\left[\begin{array}{c}
\boldsymbol{V}_{-i}^{T} \\
\boldsymbol{V}_{+i}^{T}
\end{array}\right]
$$

Defining $\varepsilon_{i}=\left[\begin{array}{llll}0 & 0 \ldots 010 \ldots 0\end{array}\right]^{T}$, with the $i$ th component as 1 , and other components as $0 \mathrm{~s}$. The estimate of the reconstructed signal is calculated from

$$
z_{i} \approx \varepsilon_{i}^{T} \boldsymbol{V}_{\alpha}\left(\boldsymbol{V}_{i}^{T} \boldsymbol{V}_{i}\right)^{-1} \boldsymbol{V}_{i}^{T} \boldsymbol{x}_{i}
$$

\section{B. SOMNN}

A SOMNN is a competitive learning network [7]. An input data vector, $\boldsymbol{x}=\left[x_{1}, x_{2}, \ldots, x_{I}\right] \in \mathfrak{R}^{I \times 1}$, with $I$ variables (sensors), is associated with a reference vector, $\boldsymbol{r}_{i} \in \mathfrak{R}^{I \times 1}$, which is often randomly initiated to give each neuron a displacement vector in the input space. For each sample of $\boldsymbol{x}(t), \boldsymbol{r}_{w}(t)$ constitutes 'the winner', by seeking the minimum distance between the input vector and the reference vector, and is calculated from:

$$
\left\|\boldsymbol{x}(t)-\boldsymbol{r}_{w}(t)\right\| \leq\left\|\boldsymbol{x}(t)-\boldsymbol{r}_{i}(t)\right\| \text { for } \forall \mathrm{i} .
$$

After obtaining a 'winner', the reference vectors are updated using:

$$
\boldsymbol{r}_{i}(t+1)=\boldsymbol{r}_{i}(t)+n_{w, i}(t)\left(\boldsymbol{x}(t)-\boldsymbol{r}_{i}(t)\right)
$$

where $n_{w, i}(t)$ is a neighbourhood function, which is normally chosen as Gaussian. The reference vectors are adjusted to match the training signals, in a regression process over a finite number of steps, in order to achieve the final 'self-organizing maps'.

Based on SOMNN algorithm, for a 2-dimensional output space, the faulted signal can be reconstructed by adjusting the weight vector using a combination of its $\mathrm{k}$ nearest nodes. Firstly, an activation function is defined to measure the activation of output neuron $\mathrm{n}$ for an input vector $\boldsymbol{x}$ by using a Gaussian kernel:

$$
T(n)=\exp \left(\frac{-1}{2 \sigma_{n}^{2}}\left\|\boldsymbol{x}-\boldsymbol{r}_{n}\right\|^{2}\right)
$$

where $\sigma_{n}^{2}$ is a parameter representing the influence region of neuron $\mathrm{n}$. When the current sample of the sensor is detected as being faulty, the winning neuron for this measurement is no longer valid, and the weighting vector is estimated by considering the $k$-nearest neighbouring neurons of the corresponding winning neuron in the output space:

$$
\boldsymbol{z}_{i} \approx \sum_{m=1}^{k}\left(T_{m} \boldsymbol{r}_{i m}\right) / \sum_{m=1}^{k} T_{m}
$$

where $z_{i}$ is the estimation of the measurement, $i$ is sensor index and $m$ is the neuron index.

\section{C. $\boldsymbol{A O}$}

To formulate the problem, assume that there are a total of $J$ time samples from a system that comprises $I$ sensorswhere $M$ of the sensors are considered to be functioning normally and the remaining $(I-M)$ sensors have been identified to be at fault. To reconstruct an estimate of the measurements from the faulted sensors, it is necessary to determine a statistical relationship amongst all the sensors during normal operation.

To investigate a suitable relationship, firstly, let $\hat{\boldsymbol{x}}_{i} \in \mathfrak{R}^{M \times 1}$ denote a measurement set from $M$ non-faulted sensors and $\hat{z}_{i} \in \mathfrak{R}^{(I-M) \times 1}$ be a data set from $(I-M)$ faulted sensors: note that the measurements are taken when all the sensors are considered to be operating normally. For simplicity, it is assumed that the sensor measurements possess a linear correlation, hence, $\hat{\boldsymbol{z}}_{j} \approx \boldsymbol{B} \hat{\boldsymbol{x}}_{j}+\boldsymbol{C}$, for $j=0,1, \cdots, J-1 \quad, \quad$ where $\quad \hat{z}_{i}=\left(\begin{array}{c}\hat{z}_{j, 0} \\ \vdots \\ \hat{z}_{j, I-M-1}\end{array}\right) \in \mathfrak{R}^{(I-M) \times 1}$,
$\boldsymbol{B}=\left[\begin{array}{c}\boldsymbol{b}_{0}{ }^{T} \\ \vdots \\ \boldsymbol{b}_{I-M-1}^{T}\end{array}\right] \in \mathfrak{R}^{(I-M) \times M}, \boldsymbol{b}_{\boldsymbol{j}} \in \mathfrak{R}^{M \times 1}$, and $\boldsymbol{C} \in \mathfrak{R}^{(I-M) \times 1}$. To 'optimally' reconstruct the data, the following $l_{2}$-norm error is proposed to be minimized:

$$
\min _{\boldsymbol{B}, \boldsymbol{C}} J(\boldsymbol{B}, \boldsymbol{C})=\sum_{j=0}^{J-1}\left\|\boldsymbol{B} \hat{\boldsymbol{x}}_{j}+\boldsymbol{C}-\hat{\boldsymbol{z}}_{j}\right\|_{2} .
$$

This problem can be simplified by letting $\boldsymbol{A}=\left[\begin{array}{ll}\boldsymbol{B} & \boldsymbol{C}\end{array}\right]$ and $\boldsymbol{x}_{j}=\left[\begin{array}{c}\hat{\boldsymbol{x}}_{j} \\ 1\end{array}\right] \in \mathfrak{R}^{(M+1) \times 1}$, then the equivalent solution is obtained from the following minimization problem:

$$
\min _{\boldsymbol{A}} J(\boldsymbol{A})=\sum_{j=0}^{J-1}\left\|\boldsymbol{A} \boldsymbol{x}_{j}-\hat{z}_{j}\right\|_{2}^{2}
$$

The solution is then simply $\boldsymbol{A}^{*}=\left[\begin{array}{c}\left(\boldsymbol{a}_{0}^{*}\right)^{T} \\ \vdots \\ \left(\boldsymbol{a}_{I-M-1}^{*}\right)^{T}\end{array}\right]$, where

$$
\boldsymbol{a}_{k}^{*}=\left(\boldsymbol{X} \boldsymbol{X}^{T}\right)^{-1} \boldsymbol{X} \tilde{\boldsymbol{z}}_{k},
$$

$\boldsymbol{X}=\left[\boldsymbol{x}_{0}, \cdots, \boldsymbol{x}_{J-1}\right], \tilde{z}_{k}=\left(\begin{array}{c}\hat{z}_{0, k} \\ \vdots \\ \hat{z}_{J-1, k}\end{array}\right)$ for $k=0, \cdots,(I-M-1)$, and $\operatorname{rank}\left(\boldsymbol{X} \boldsymbol{X}^{T}\right)=M$. 


\section{International Journal of Advances in Computer Science \& Its Applications}

Volume 6 : Issue 1

[ISSN 2250-3765]

Publication Date : 18 April, 2016

After calculating the optimal matrix $\boldsymbol{A}^{*}$, the data of the remaining $(I-M)$ sensors can be estimated from:

$$
z_{i} \approx \boldsymbol{A}^{*} \boldsymbol{x}_{i}, \text { for } i=0,1, \cdots, I-1 .
$$

since an analytical formula for obtaining the 'optimal matrix' in (13) is derived a-priori, no iterative numerical optimization procedure is required [5].

\section{Case Study}

To provide an illustrative focus to the study, a group of six burner tip temperature sensors, which are used to monitor the combustion system on an industrial gas turbine system, is investigated, as illustrated in Fig. 1.

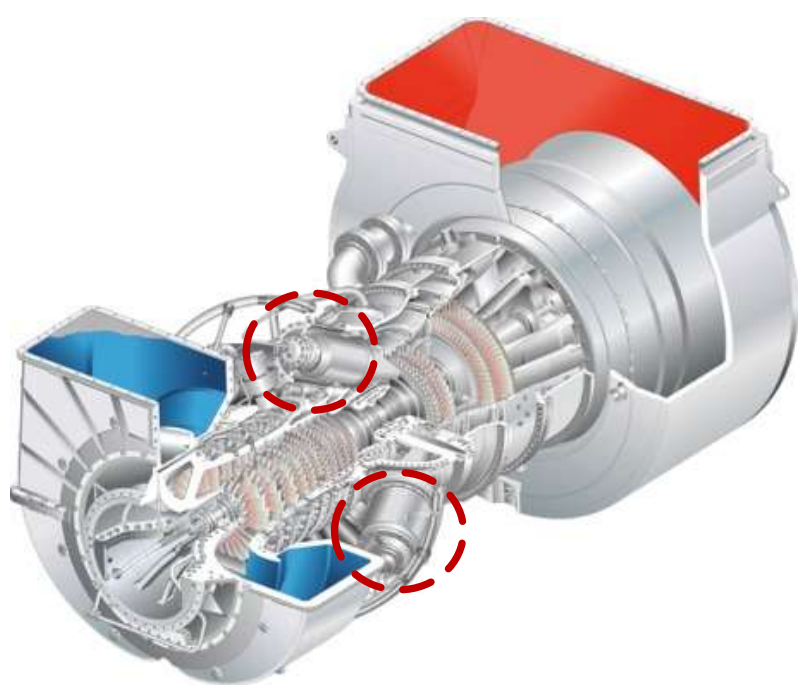

Figure 1. Industrial gas turbine and its combustion system

To evaluate the reconstruction performance of (6), (10) and (14), the $l_{2}$-norm relative reconstruction error, $E$, is defined as follows,

$$
E=\frac{\sqrt{\sum_{i=1}^{I}\left\|z_{i}-\boldsymbol{x}_{i}\right\|_{2}^{2}}}{\sqrt{\sum_{i=1}^{I}\left\|x_{i}\right\|_{2}^{2}}} .
$$

In this case, normal operational measurements from the burner tip temperature sensors are as shown in Fig. 2. The first 300 minutes are used as training data, and signal for Sensor 6 from 301 to 1440 minutes is estimated.

The original and estimated measurements are shown in Figs. 3(a), (b) and (c) for the three approaches. The $l_{2}$ norm relative prediction errors are $0.13 \%, 0.19 \%$ and $0.12 \%$ respectively, for the $\mathrm{PCA}, \mathrm{SOMNN}$ and AO based approaches, signifying that the reconstruction bears an exce llent correspondence with the real measurements. PCA and AO based approaches give very similar reconstruction results.

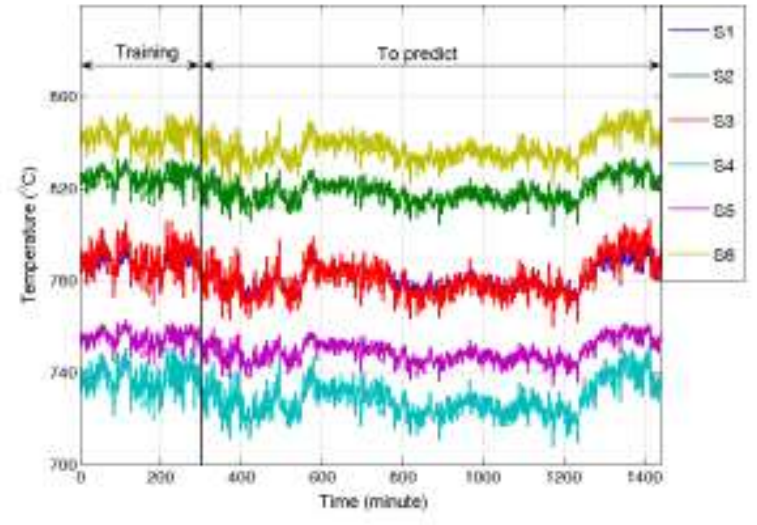

Figure 2. Signal reconstruction testing example ( $\mathrm{S}=$ Sensor $)$

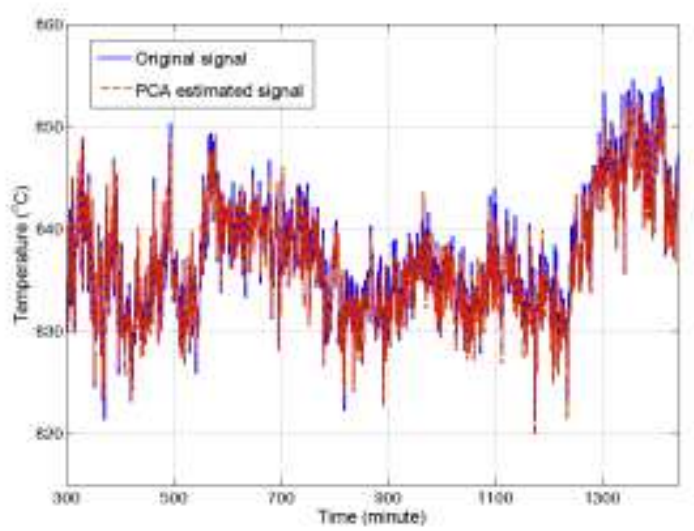

(a)

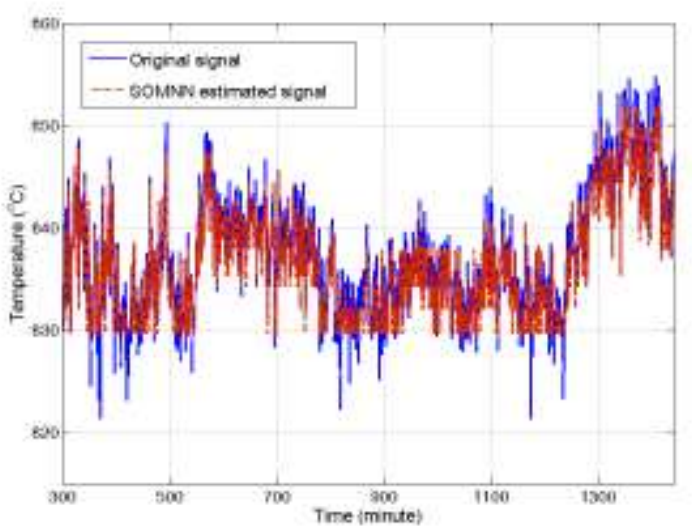

(b)

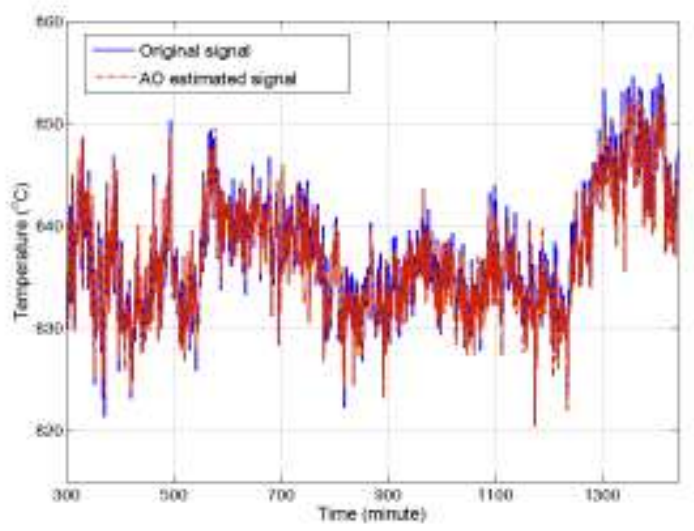

(c)

Figure 3. Reconstructed signal based on (a) PCA; (b) SOMNN and (c) AO 
SOMNN based approach is subject to a relatively poor performance - this is attributed to using a limited subset of training data, and could be enhanced by a longer prior training period.

Since AO gives the best performance from the testing example, the same principle is applied to the experimental trial to detect a sensor failure, as shown in Fig. 4. The faulted signal is reconstructed based on the AO algorithm, as shown in Fig. 5. From the results it can be seen that from the onset of the 'fault period', the reconstructed data follows the normal trend (given by the behaviour of the other sensors) very reliably, and could therefore be used in place of the erroneous measurements in the short term.

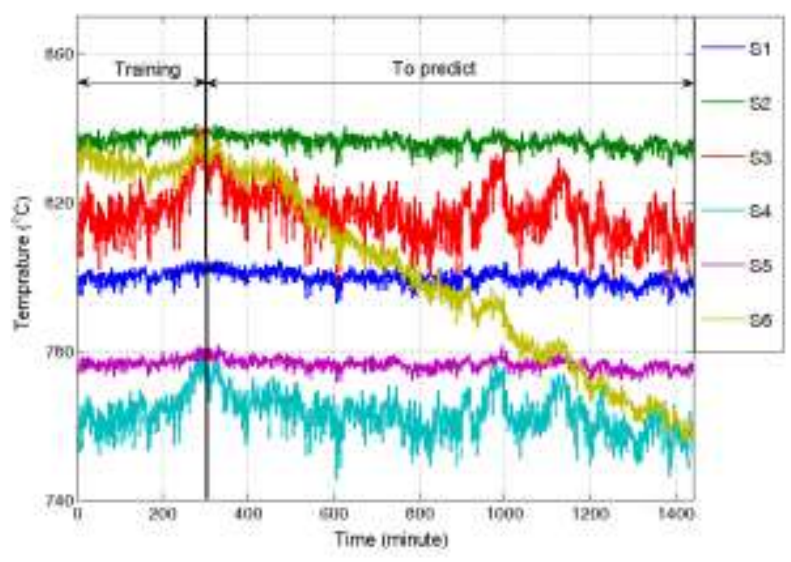

Figure 4. Faulted signal reconstruction temperature measurements ( $\mathrm{S}=$ Sensor)

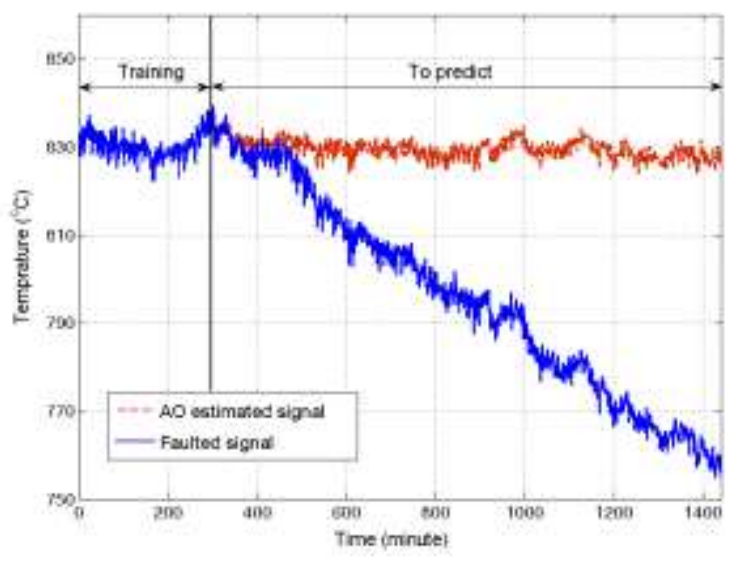

Figure 5. Faulted signal reconstruction based on AO algorithm

It should be noted that these techniques can also be readily adapted to provide expected outputs from each sensor in a group, which can then be compared to the realtime measurements, and thereby provide a further simple mechanism for detecting unexpected sensor behaviour.

\section{Iv. Conclusion}

In this paper, three approaches are investigated for sensor measurement reconstruction in an industrial system: 1) PCA based missing value method; 2) SOMNN based technique; and 3) an AO method. The applied approaches are shown to be capable to and reconstructing sensor faults successfully. The efficacy of the presented approaches has been proved through the use of results from experimental trials. Signal reconstruction techniques can be used for fault detection and reconstruct faulted sensor signals, and thereby facilitate improved unit availability. Although the current study focuses on burner tip temperatures on industrial gas turbines, ultimately, the underlying principles are readily transferable to other complex industrial systems.

\section{Acknowledgment}

The authors would like to thank Siemens Industrial Turbomachinery, Lincoln, U.K., for providing research support and access to real-time data to support the research outcomes.

\section{References}

[1] Y. Zhang, C.M. Bingham, M. Gallimore, Z. Yang, and J. Chen. "Sensor Fault Detection for Industrial Systems Using a Hierarchical Clustering-based Graphical User Interface," In Proc. of IEEE Conf. on Multisensor Fusion and Integration for Intelligent Systems (MFI), Hamburg, Germany, Sep. 2012, pp. 389-394.

[2] Y. Zhang, C.M. Bingaham, Z. Yang, M. Callimore and P. Stewart. "Applied Sensor Fault Detection and Identification Using Hierarchical Clustering and SOMNNs, with Faulted-Signal Reconstruction," In Proc. of 15th Int. Symp. on Mechatronika, Prague, Czech Republic, Dec. 2012, pp. 107-113.

[3] Y. Zhang, C.M. Bingham, and M. Gallimore."Fault Detection and Diagnosis Based on Extensions of PCA," Advances in Military Technology, vol. 8, no. 2, 2013, pp. 27- 41.

[4] Y. Zhang, C.M. Bingham, M. gallimore, Z. Yang, and J. Chen. "Sensor fault detection and diagnosis based on SOMNNs for steadystate and transient operation," In Proc. of IEEE Int. Conf. on Computational Intelligence and Virtual Environments for Measurement Systems and Applications (CIVEMSA), Milan, Italy, Jul. 2013. pp. 110-115.

[5] Z. Yang, B.W. Ling, and C.M. Bingham, "Fault detection and signal reconstruction for increasing operational availability of industrial gas turbines," Measurement, vol. 46 ,no. 6, 2013, pp. 1938-1946.

[6] H. Martin, T. Naes, Multivariate Calibration, John Wiley and Sons, New York, 1989.

[7] T. Kohonen, Self-Organizing Maps, Heidelberg: Springer Verlag, 1995. 